\title{
ARTICLE OPEN \\ Metagenomic and metatranscriptomic analysis of saliva reveals disease-associated microbiota in patients with periodontitis and dental caries
}

\author{
Daniel Belstrøm ${ }^{1}$, Florentin Constancias ${ }^{2}$, Yang Liu ${ }^{2}$, Liang Yang (D) $^{2}$, Daniela I. Drautz-Moses ${ }^{2}$, Stephan C. Schuster ${ }^{2}$, \\ Gurjeet Singh Kohli ${ }^{2}$, Tim Holm Jakobsen ${ }^{3}$, Palle Holmstrup ${ }^{1}$ and Michael Givskov ${ }^{2,3}$
}

The taxonomic composition of the salivary microbiota has been reported to differentiate between oral health and disease. However, information on bacterial activity and gene expression of the salivary microbiota is limited. The purpose of this study was to perform metagenomic and metatranscriptomic characterization of the salivary microbiota and test the hypothesis that salivary microbial presence and activity could be an indicator of the oral health status. Stimulated saliva samples were collected from 30 individuals (periodontitis: $n=10$, dental caries: $n=10$, oral health: $n=10$ ). Salivary microbiota was characterized using metagenomics and metatranscriptomics in order to compare community composition and the gene expression between the three groups. Streptococcus was the predominant bacterial genus constituting approx. 25 and 50\% of all DNA and RNA reads, respectively. A significant disease-associated higher relative abundance of traditional periodontal pathogens such as Porphyromonas gingivalis and Filifactor alocis and salivary microbial activity of $F$. alocis was associated with periodontitis. Significantly higher relative abundance of caries-associated bacteria such as Streptococcus mutans and Lactobacillus fermentum was identified in saliva from patients with dental caries. Multiple genes involved in carbohydrate metabolism were significantly more expressed in healthy controls compared to periodontitis patients. Using metagenomics and metatranscriptomics we show that relative abundance of specific oral bacterial species and bacterial gene expression in saliva associates with periodontitis and dental caries. Further longitudinal studies are warranted to evaluate if screening of salivary microbial activity of specific oral bacterial species and metabolic gene expression can identify periodontitis and dental caries at preclinical stages.

npj Biofilms and Microbiomes (2017)3:23; doi:10.1038/s41522-017-0031-4

\section{INTRODUCTION}

Periodontitis and dental caries are the two most prevalent oral diseases and the primary causes of tooth loss in the western world. ${ }^{1,2}$ At present, periodontitis and dental caries are mostly diagnosed at late stages of disease, often leading to costly and invasive dental treatment. Therefore, new diagnostic approaches capable of identifying periodontitis and dental caries at preclinical stages, favoring preventive treatment strategies, are urgently needed.

The oral cavity harbors a diverse microbiota comprising more than 700 unique bacterial species. ${ }^{3}$ The microbiota plays a pivotal role in maintenance of oral homeostasis, as various oral habitats are colonized by characteristic bacterial community profiles organized in local biofilms. ${ }^{4}$ However, ecological changes, for example in relation to increased sugar intake, insufficiently performed oral hygiene or fluctuations in the immune response can induce structural $\left.\right|^{5-7}$ and functional alterations ${ }^{8-10}$ of local oral biofilms. Such alterations may in turn change the relation between the host and the resident microbiota from symbiosis to dysbiosis, thereby fueling initiation and progression of periodontitis and dental caries.
Saliva is the biological fluid of the oral cavity which is critical for maintenance of oral and general health. ${ }^{11}$ Therefore, saliva has been intensively investigated for candidate biomarkers associated with oral health and disease. ${ }^{12,13}$ Saliva is sterile when entering the oral cavity, ${ }^{14}$ but when sampled, saliva contains a diverse microbiota. ${ }^{15}$ In healthy oral conditions, the composition of the salivary microbiota is different from that of supragingival and subgingival biofilms. ${ }^{16}$ On the other hand, the presence of specific bacterial species in saliva such as Porphyromonas gingivalis and Streptococcus mutans has been reported in individuals with periodontitis and dental caries, respectively. ${ }^{17,18}$ Essentially, these findings suggest that bacteria from local periodontitis and caries lesions may be spilled-over and dispersed into saliva. However, it remains unclear if dispersed bacteria remain metabolically active as they are translocated from the local ecological niche of the biofilms to saliva, which possesses different ecological properties.

So far, only a few studies have reported higher expression of specific bacterial genes to associate with dental caries. ${ }^{19,20}$ In addition, the potential of using comprehensive metatranscriptomic microbial analysis of saliva to identify possible biomarkers has not yet been evaluated.

\footnotetext{
${ }^{1}$ Section for Periodontology and Oral Microbiology, Department of Odontology, Faculty of Health and Medical Sciences, University of Copenhagen, Copenhagen, Denmark ${ }^{2}$ Singapore Centre for Environmental Life Sciences Engineering, Nanyang Technological University Singapore, Singapore, Singapore and ${ }^{3}$ Costerton Biofilm Center, Department of Immunology and Microbiology, Faculty of Health and Medical Sciences, University of Copenhagen, Copenhagen, Denmark

Correspondence: Daniel Belstrøm (dbel@sund.ku.dk)

Daniel Belstrøm and Florentin Constancias contributed equally to this work.
}

Received: 20 February 2017 Revised: 3 August 2017 Accepted: 1 September 2017

Published online: 02 October 2017 
The aims of the present study were to employ metagenomic and metatranscriptomic analyses to (i) characterize the diversity and community composition of the salivary microbiota, (ii) to utilize these data to determine the microbial activity (DNA/RNA ratio) of each taxon identified and, (iii) to identify differentially expressed microbial genes. The main hypothesis was that the taxonomic composition, as well as microbial activity could be an indicator of the oral health status, and possible differences might potentially be used as proxy biomarkers of oral health and disease.

\section{RESULTS}

General information

From a total of 30 saliva samples $768.62 \mathrm{M}$ (mean $25.62 \mathrm{M}$; range $14.61 \mathrm{M}-32.64 \mathrm{M}$ ) DNA sequences and $981.48 \mathrm{M}$ (mean $32.72 \mathrm{M}$; range $26.40 \mathrm{M}-38.44 \mathrm{M}$ ) RNA sequences were generated out of which $274.17 \mathrm{M}$ (DNA; mean 9.14 M: range $2.58 \mathrm{M}-18.52 \mathrm{M}$ ) and 148.49 (RNA; mean $4.50 \mathrm{M}$; range $2.93 \mathrm{M}-9.11 \mathrm{M}$ ) passed quality control (Supporting material Table S1).

At species level, a significantly higher a-diversity (observed species) was recorded by metagenomics compared to metatranscriptomic analysis (Figs. 1a-b) $(p<0.05)$. However, no difference in a-diversity was observed between groups using either metagenomics or metatranscriptomic approaches. Metagenomic analysis yielded a significantly different $\beta$-diversity between groups $\left(p=0.027, R^{2}=0.12\right)$, whereas an insignificantly different $\beta$-diversity was recorded when employing metatranscriptomic analysis ( $p=0.11, R^{2}=0.10$ ) (Fig. 1c).

A total of 810 to 894 microbial KEGG Orthologs (KO) was identified in salivary microbial metagenomes and metatranscriptomes. These KO were mainly associated to Ribosome, Carbon metabolism, $\mathrm{ABC}$ transporter, and Purine metabolism pathways (Supporting material Fig S1A). Within the metabolism category salivary microbiomes were dominated by formate C-acetyltransferase, enolase, 5-methyltetrahydropteroyltriglutamate--homocysteine methyltransferase, and pyruvate oxidase (Supporting material Fig S2A). In all cases, a significantly lower number of KEGG KO and Pathways were identified in the salivary metagenomes as compared to the microbial metatranscriptome $(p<0.05$, Supporting material Figs S1B, S2B). In order to characterize the overall community differences in terms of KEGG pathways and KO, Bray-Curtis overall dissimilarities were constructed and no significant discrimination of healthy and disease groups were observed (PERMANOVA $p>0.05$ in all case, Supporting material Figs S1 C-D; Fig S2 C-D).
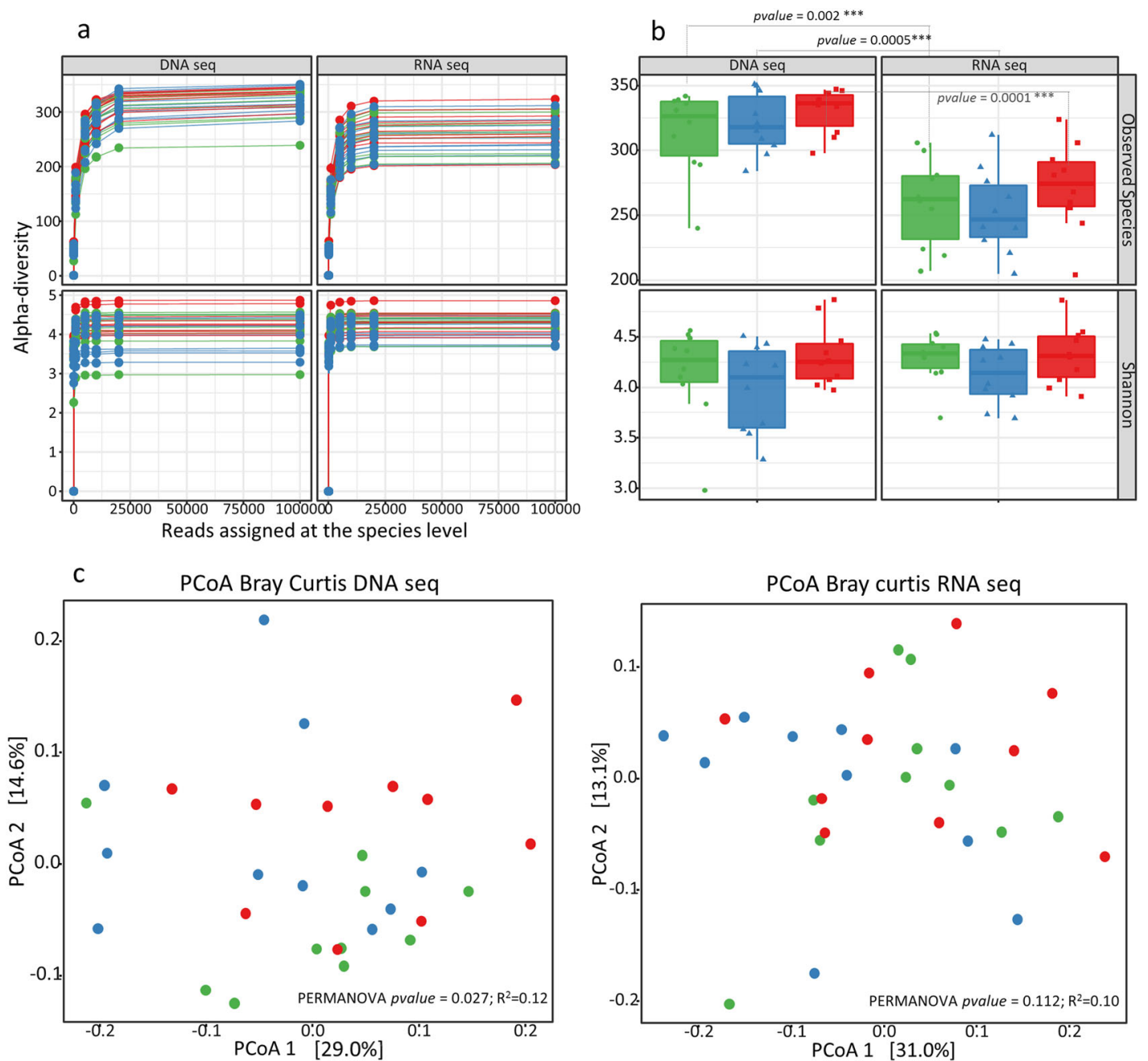

Fig. 1 Microbial diversity. a: Rarefaction curves expressed as number of reads assigned at species level ( $x$-axis) and $\alpha$-diversity (observed Species and Shannon index, $y$-axis) of salivary microbiota characterized using metagenomics and metatranscriptomics. $\mathbf{b}$ : Distribution of $\alpha$ diversity among groups presented as boxplot (median, lower/upper quartile and standard deviation). c: $\beta$-diversity determined by Bray-Curtis dissimilarity and plotted using PCoA. Sample denotation: green: healthy controls, blue: dental caries, red: periodontitis 
Identification of microbial taxa from salivary metagenomes and metatranscriptome

A total number of 12 bacterial phyla, 123 bacterial genera, and 351 bacterial species were identified from the salivary metagenome with a mean number per sample of 11 phyla (10-12), 115 genera (95-123), and 321 species (240-351). Metatranscriptomic bacterial identification yielded a total number of 12 bacterial phyla, 101 bacterial genera, and 234 bacterial species with a mean number per sample of 10 phyla (8-12), 101 genera (79-120), and 262 species (204-324).

Twenty most abundant bacterial genera based on metagenomic and metatranscriptomic bacterial identifications are displayed in Fig. 2a. As seen in Fig. 2a, the three most predominant genera based on both identification methods were Streptococcus, Prevotella, and Veillonella constituting approx. $70 \%$ of all assigned DNA and RNA reads. Streptococcus was significantly more abundant based on metatranscriptomic identification (50\%) than metagenomic identification $(25 \%, p<0.05)$.

The predominant 20 bacterial species based on metagenomic and metatranscriptomic bacterial identifications are displayed in Fig. 2b. While the salivary metagenomes were dominated by equal proportions of Streptococcus $s p$. and Rothia $s p$. the salivary metatranscriptomes were characterized by high proportions of Streptococcus sp. but very low proportions of Rothia $s p$.

Salivary abundance of specific oral bacterial species associates with periodontitis and dental caries

From a total of 391 bacterial species identified using metagenomic analysis, 264 constituted the core salivary microbiome, and the majority of bacterial species $(84.9 \%)$ were shared between samples from periodontitis patients, dental caries patients, and orally healthy controls (Fig. 3a). In addition, 32 periodontitisassociated, 13 caries-associated, and 11 oral health-associated microbial species were identified based on relative abundance of assigned DNA reads ( $p<0.05$, Fig. $3 \mathrm{~b}$ ).

Based on metatranscriptomic analysis a total of 105 bacterial species constituting $76.7 \%$ of the total relative abundance were shared between the three groups (Fig. 3c). In addition, 22 periodontitis-associated, 14 caries-associated, and 8 oral healthassociated microbial species were identified based on relative abundance of assigned RNA reads ( $p<0.05$, Fig. $3 d$ ).

Traditional periodontal pathogens such as $P$. gingivalis, Tannerella forsythia, Parvimonas micra, and F. alocis and cariogenic bacteria, including $S$. mutans and Lactobacillus $s p$. were identified with significantly higher relative abundance in samples from periodontitis and dental caries patients, respectively, based on both metagenomic and metatranscriptomic approaches (Figs. 3b, d).

Microbial activity of specific oral bacterial species associates with oral health and disease

Based on comparison of RNA/DNA ratio at phylum level Firmicutes was the only taxon expressing a positive RNA/DNA ratio in all groups, while the majority of remaining 12 phyla were recorded with negative RNA/DNA ratios (Fig. 4a). A total of 23 bacterial species were identified with significantly different RNA/DNA ratios between groups $(p<0.05)$ (Fig. 4b).

Salivary metabolic gene expression in periodontitis, caries, and oral health

Differential gene expression analysis (i.e., metatranscriptomic data) allowed the identification of 15 differentially expressed $\mathrm{KO}$ between periodontitis patients and healthy controls (2 periodontitis-associated and 13 health-associated), out of which ten belonged to the KO metabolism category and five belonged to the KO Environmental Processing Information category $(p<$
0.05 , Fig. 5a). The majority of $\mathrm{KO}$, which was down-regulated in periodontitis compared to orally healthy controls, was involved in carbohydrate metabolism (K00917, K04072, K12308, K02793, K01788, K12373, K02775, and K01443). Four differentially expressed $\mathrm{KO}$ were recorded between caries patients and healthy controls (two caries-associated and two health-associated), from which three belonged to the KO metabolism category and one belonged to the $\mathrm{KO}$ environmental processing information category $(p<0.05$; Fig. 5b). The two KO, which were upregulated in caries was involved in glycan biosynthesis (K07260) and carbohydrate metabolism (K00869), whereas lipid metabolism (K00105) was up-regulated in orally healthy controls compared to caries patients (Fig. 5b).

\section{DISCUSSION}

The purpose of this study was to characterize and compare salivary microbiota in oral health and disease using metagenomic and metatranscriptomic approaches. The primary finding was that both metagenomic and metatranscriptomic analyses identified higher salivary abundance of specific oral bacterial species in periodontitis and dental caries patients. Furthermore, salivary expression of specific metabolic genes was significantly different in patients with periodontitis and caries compared to healthy controls, and high activity (RNA/DNA ratio) of $F$. alocis and Neisseria sp. was associated with periodontitis. To the best of our knowledge, this is the first study to perform comprehensive simultaneous metagenomic and metatranscriptomic characterization of the salivary microbiota.

Streptococcus was identified as the most predominant bacterial genus by use of both metagenomic and metatranscriptomic analyses (Figs. 2a-b). These findings are in line with 16Smetabarcoding and metaproteomic analyses performed on the same samples, ${ }^{17,21}$ and underline the importance of Streptococcus in the composition of the salivary microbiota. Moreover, metagenomic and metatranscriptomic analyses yielded significant differences in characterization of the salivary microbiota (Figs. $2 a-b)$. Remarkably, this could be acknowledged at genus level where Streptococcus accounted for approx. 25\% of all assigned DNA reads compared to $50 \%$ of all assigned RNA reads. This finding was further supported, as phylum Firmicutes, which includes the genus Streptococcus, was the most active bacterial phylum in saliva as determined by the RNA/DNA ratio (Fig. 4a). Interestingly, this observation aligns with metagenomic and metatranscriptomic data extracted from a batch of supragingival samples, in which major differences in bacterial metagenomic and metatranscriptomic profiles were observed. ${ }^{5,8}$ Thus, this observation suggests that some bacterial genera, including Streptococcus may constitute the more active part of the salivary microbiota, while other members of the salivary microbiota, for example Rothia sp. might be largely inactive.

Based on relative abundance of the taxa at metagenomic level, multiple periodontitis-associated and caries-associated bacterial species were identified (Fig. 3b). These findings are in line with previous studies employing various contemporary molecular methods for bacterial identification in saliva, ${ }^{17,18,20,22-24}$ including 16S-metabarcoding identification performed on the same saliva samples. ${ }^{17}$ Subgingival colonization of bacterial species such as $P$. gingivalis, $T$. forsythia, $P$. micra, and $F$. alocis is considered strongly associated with periodontal disease activity. ${ }^{6,25,26}$ Furthermore, S. mutans and Lactobacillus sp. are considered key etiological agents in the cariogenic supragingival biofilm based on their proficient capability of sugar fermentation. ${ }^{7,27}$ These specific and well-known disease-associated bacterial species were identified in higher relative abundance in saliva samples from patients with periodontitis and dental caries. Essentially, this finding reinforces the concept that pathogenic bacteria are shed from local periodontitis and 

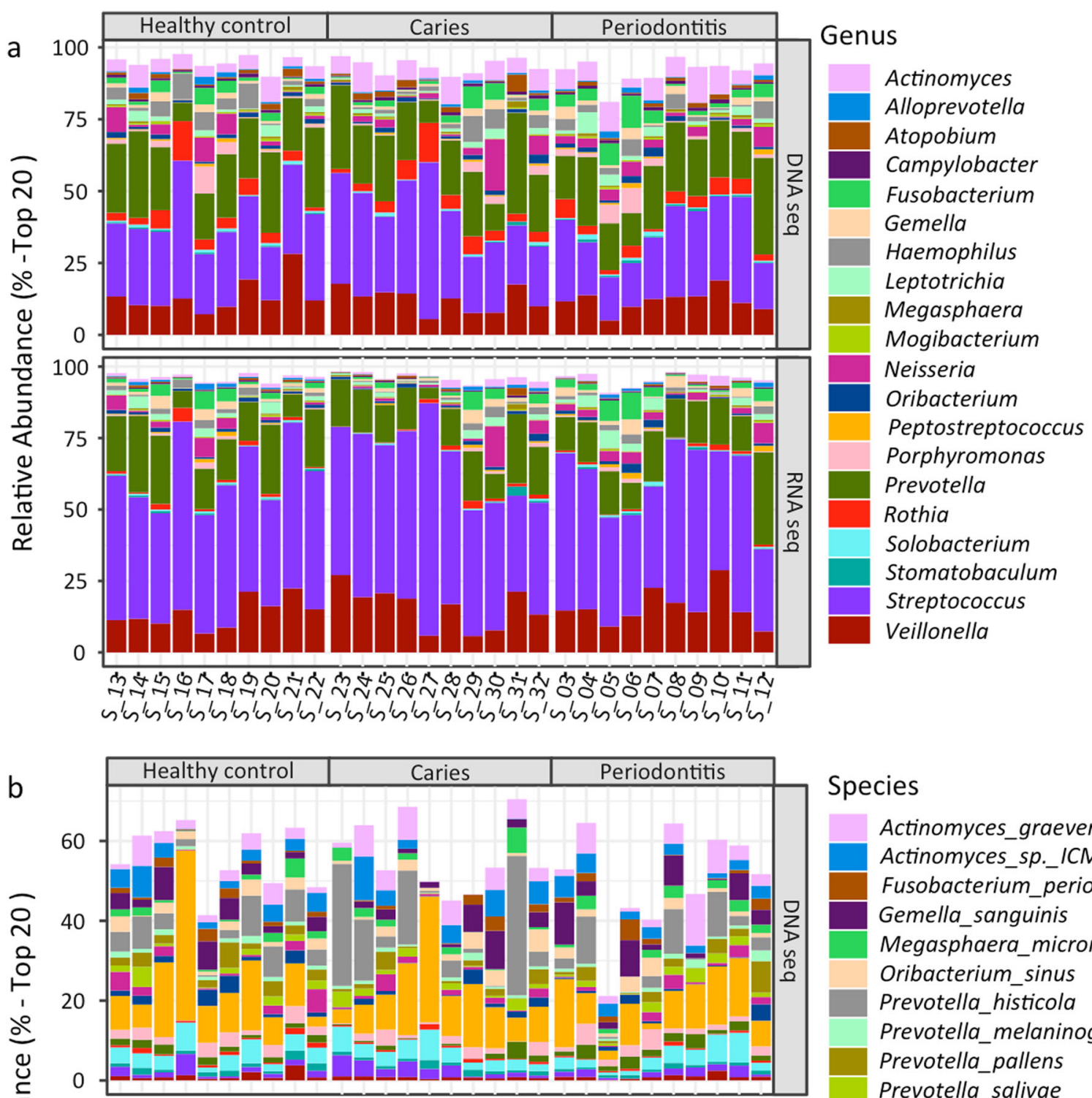

Species

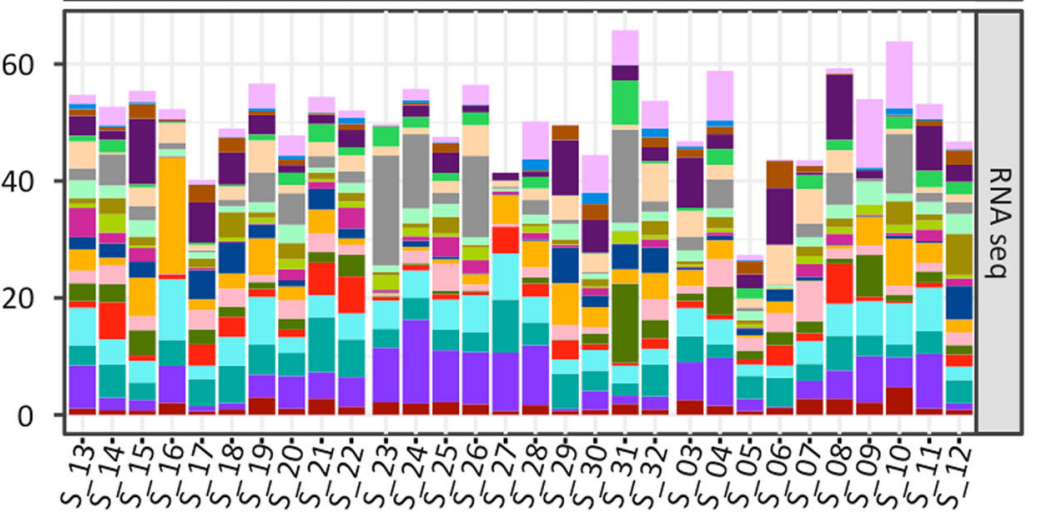

Actinomyces_graevenitzii

Actinomyces_sp._ICM47

Fusobacterium_periodonticum

Gemella sanguinis

Megasphaera_micronuciformis

Oribacterium_sinus

Prevotella_histicola

Prevotella_melaninogenica

Prevotella_pallens

Prevotella_salivae

Prevotella_sp._C561

Prevotella_sp._oral_taxon_473

Rothia_mucilaginosa

Solobacterium_moorei

Stomatobaculum_longum

Streptococcus_mitis

Streptococcus_parasanguinis

Streptococcus_pneumoniae

Streptococcus salivarius

Veillonella_sp._DORA_A_3_16_22

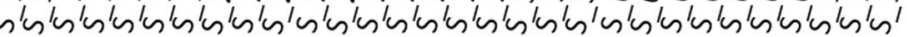

Fig. 2 Relative abundance. a: Top 20 predominant microbial genera. b: Top 20 predominant microbial species based on metagenomic and metatranscriptomic analyses

caries lesions into saliva. This may increase salivary abundance of these bacteria in patients with periodontitis and dental caries. However, low levels of $P$. gingivalis and S. mutans are also occasionally identified in saliva from orally healthy individuals, ${ }^{28,29}$ indicating that in some individuals these bacteria may be part of the resident oral microbiota.
The salivary microbiota has been reported to primarily consist of bacteria shed from the tongue, throat and tonsils. ${ }^{16}$ The turnover time of the salivary microbiota is extremely high due to continuous swallowing. ${ }^{15}$ Bacterial activity is ultimately determined by the level of substrates and local environmental conditions. $^{30,31}$ Thus, bacterial activity of the salivary microbiota 

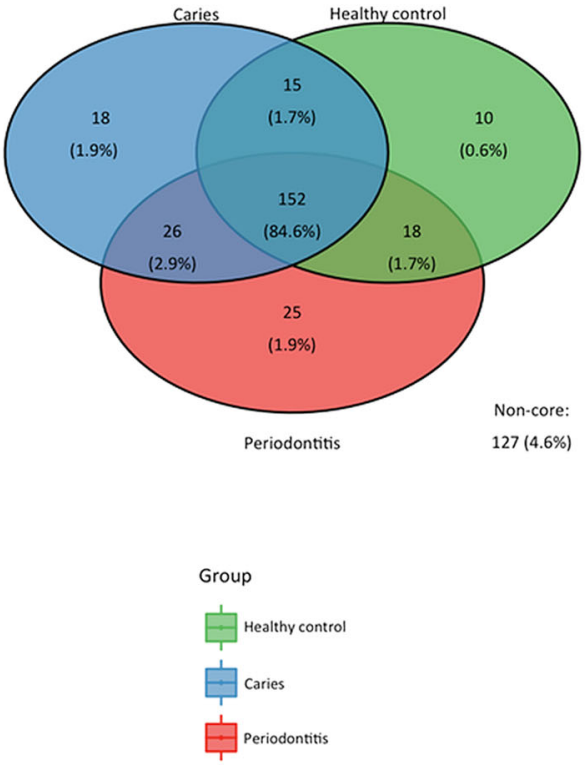

C

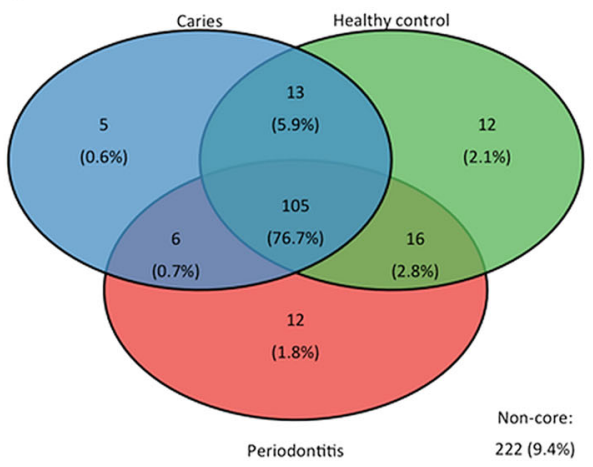

b

Oribacterium_parvum Streptococcus_pseudopneumonia Candidatus_Saccharibacteria_oral_toxon_TM7X Candidatus_Saccharibacteria_bacterium_GW2011_GWC2__44_17 Streptocaccus_oralis Escherichia_coli Streptococcus_sp._oral_taxon_os Streptococcus_sp._oral_toxon 056 Fusobacterium_nucleatum Porphyromonas_endodontalis Prevotella intermedia
Tannerella forsythia Actinomyces_dentalis filifactor_alocis Porphyromonas gingivalis Treponema denticol Parvimonas micro sibacterium_sp...CMSO Treponema_socransk Eubacterium_nodatum
Actinomyces johnsoni Eubacterium saphenu Fretibacterium fastidiosum reponema_lecithinolyticum Prevotella_baronio Treponema vincenti Treponema_maltophilum Campylobacter_rectus Treponema_medium Aceponema_sp. OMZ 838 sobacterium_necrophoru Actinomyces_urogenitalis Gardnerella vaginalis Gardnerella_vaginalis
Treponema pedis Prevotella falseni Mycoplasma_solivarium yces_sp._oral_taxon_448 streptococcus mutans acterium acidifacien Parascardovia_denticolens Streptococcus_sobrinus Lactobacilius_vaginal Lactobacillus fermentu Streptococcus_sp._C15 Lactobacillus_gasseri Lactobacillus_crispatus

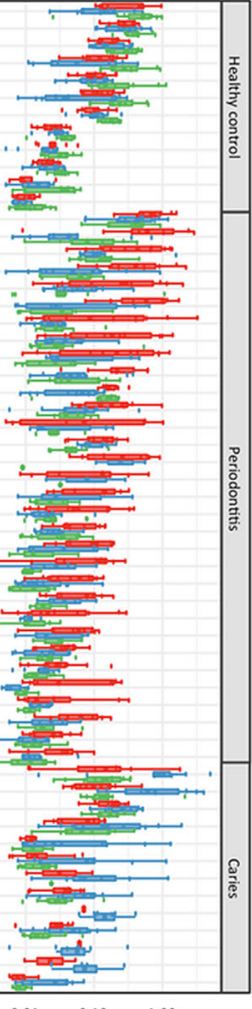

$0.01 \quad 0.10 \quad 1.00$

Proportion - \% DNA seq

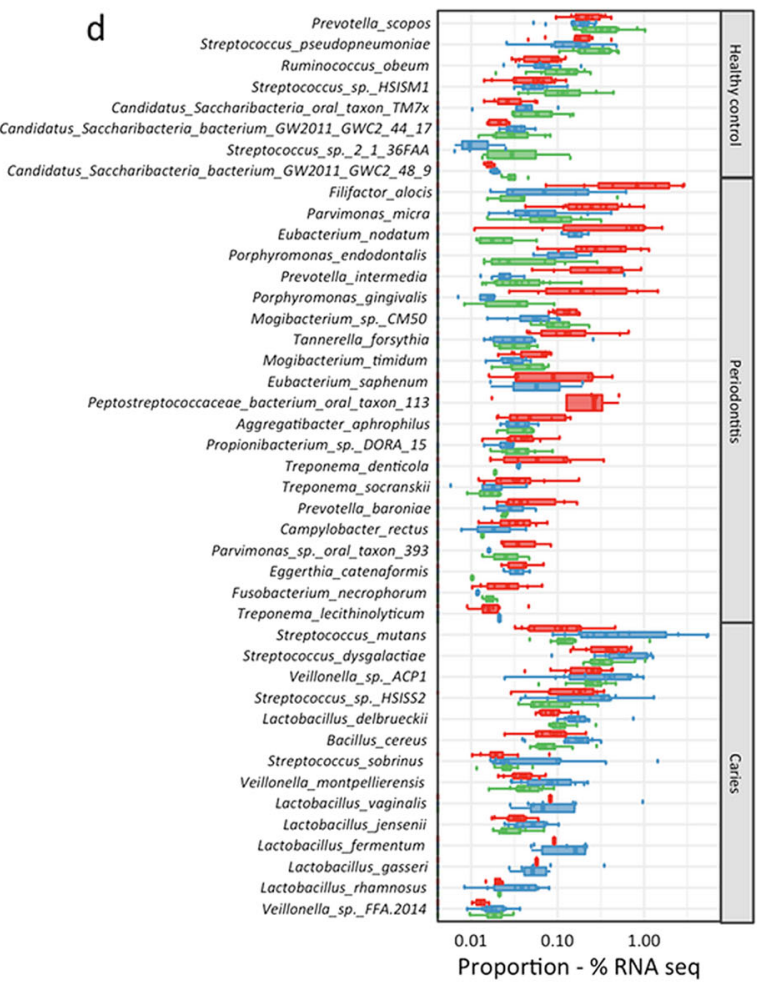

Fig. 3 Periodontitis and caries-associated salivary microbiota. a: Venn diagram displaying number and proportion of microbial species in salivary core and non-core microbiome based on metagenomic approach. b: Bacterial species with significantly higher relative abundance in oral health, dental caries or periodontitis. c: Venn diagram displaying number and proportion of microbial species in salivary core and noncore microbiome based on metatranscriptomic approach. d: Bacterial species with significantly higher relative abundance in oral health, dental caries or periodontitis. Sample denotation: green: healthy controls, blue: dental caries, red: periodontitis 
a

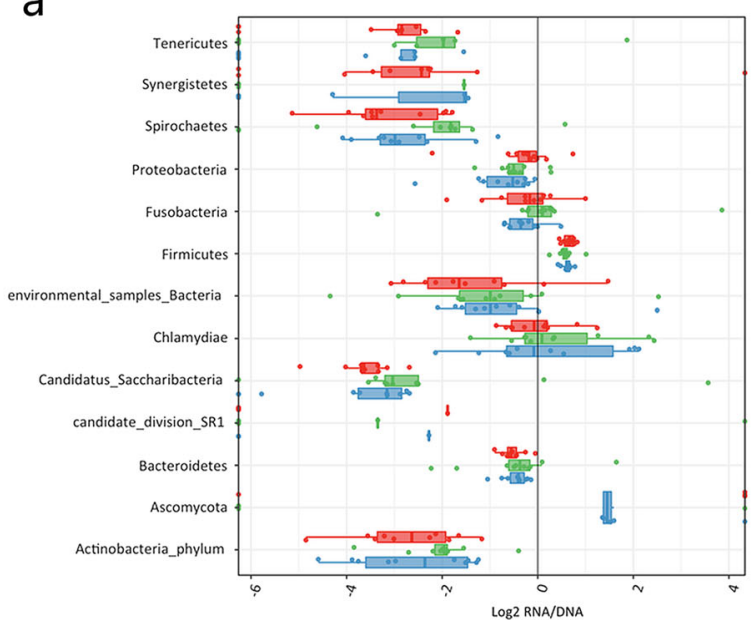

$\mathrm{b}$

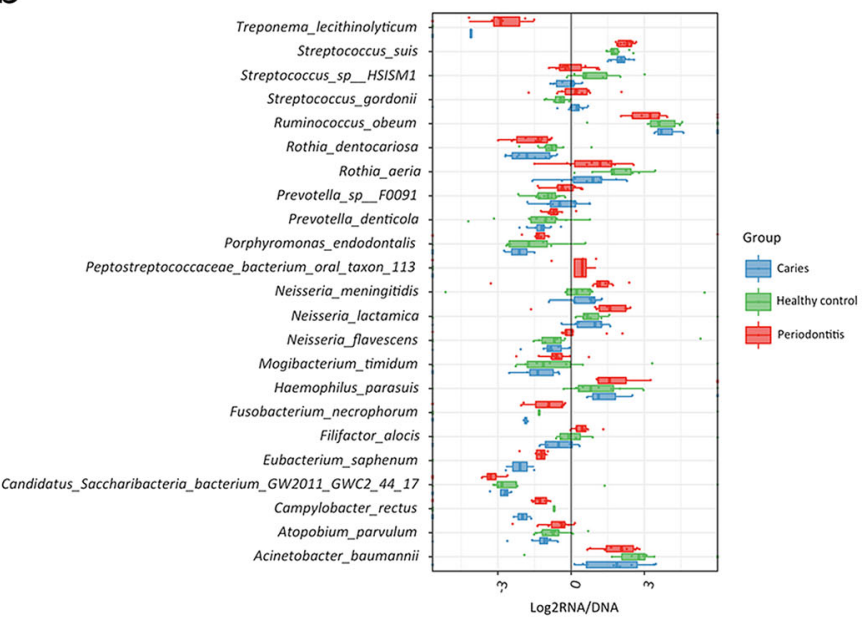

Fig. 4 Salivary microbial activity. a: Log2 RNA/DNA ratio of the 13 bacterial phyla identified in saliva across all samples. b: Bacterial species identified with significantly different log2 RNA/DNA ratios in samples from patients with periodontitis (red), dental caries patients (blue) and orally healthy controls (green)

a

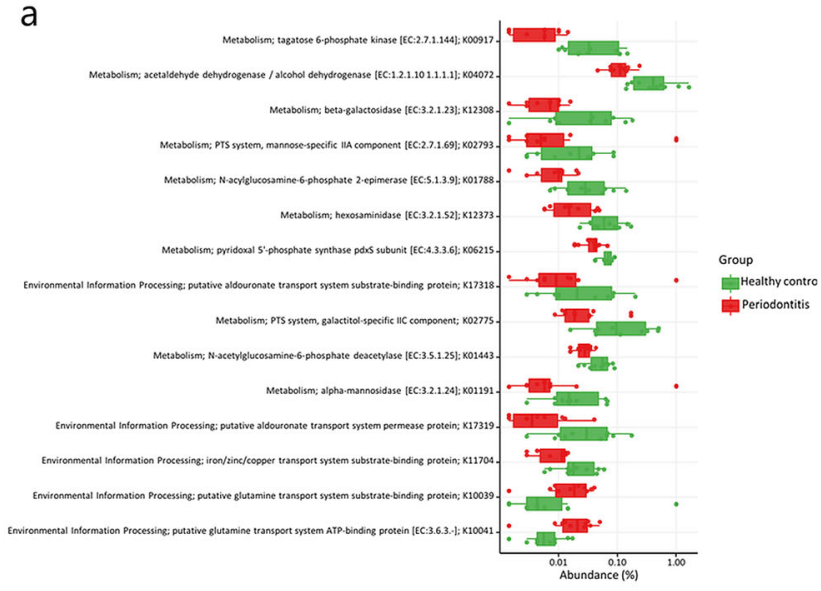

b

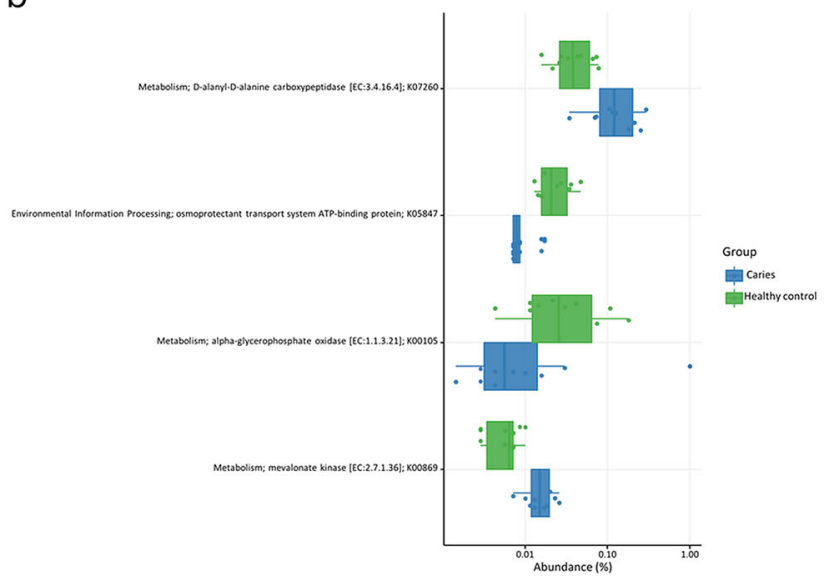

Fig. 5 Differentially expressed genes in the salivary metatranscriptome in dental health and disease. a: periodontitis patients (red) and healthy controls (green) and b: dental caries patients (blue) and healthy controls (green). Only significant differences as determined using DESeq2 negative binomial tests are shown $(p<0.05)$. KEGG orthologs $(\mathrm{KO})$ numbers and Enzyme commission numbers are reported (EC) when applicable

may relate to remnants of the phenotypic profile expressed in the local environment. Functional analysis of metatranscriptomic data highlighted significant differences in salivary gene expression in patients with periodontitis and caries compared to orally healthy controls. Saliva from orally healthy controls was associated with an up-regulation in functional activity of carbohydrate metabolism compared to periodontitis patients (Fig. 5a). Furthermore, saliva from caries patients expressed up-regulation of carbohydrate metabolism and down-regulation of lipid-metabolism compared to orally healthy controls (Fig. 5b). Thus, data from this study clearly demonstrate bacterial metabolic activity of the salivary microbiota at the community level, which is surprising, when considering the high turn-over rate of the salivary microbiota. Unfortunately, the sequencing depth was not sufficient for comparisons of species-specific metabolic activity between groups. However, as approx. 50\% of all RNA reads could be taxonomically assigned to Streptococcus species it is likely that a substantial part of salivary metabolic activity derived from Streptococcus species, which is further supported by the fact that Streptococcus species are proficient in carbohydrate metabolism. ${ }^{32}$ Notably, 16S-metabarcoding analysis of the same samples showed significantly lower relative abundance of Streptococcus species in samples from periodontitis patients compared to healthy controls, ${ }^{17}$ which suggests that metatranscriptomic profiles observed in this study probably associates with differential functional activity of salivary Streptococcus species in oral health and disease.

Taxonomic assignment of metatranscriptomic data of the salivary microbiota was employed as a proxy marker of microbial activity. Notably, all the usual suspects of traditional periodontal pathogens ( $P$. gingivalis, T. forsythia, $P$. micra, and F. alocis) and cariogenic bacteria ( $S$. mutans and Lactobacillus $s p$.) were identified with significantly higher relative abundance at metatranscriptomic level (Fig. 3d). This suggests that bacteria shed from local diseased lesions sustain microbial activity when dispersed into saliva. This is further demonstrated by the higher salivary RNA/DNA ratio of $F$. alocis and Neisseria sp. in patients with periodontitis. Consequently, higher salivary bacterial activity of the usual suspects of periodontitis ( $P$. gingivalis, $T$. forsythia, $P$. micra, and $F$. alocis) and dental caries (S. mutans and Lactobacillus $s p$.) may possess a potential for use as a biomarker of the respective diseases. Further studies with additional sequencing depth and larger sample size (including supra and subgingival samples) 
would allow the comparison of salivary and local diseased lesions' microbiota. This would further strengthen the finding of the present study.

In conclusion, this study is the first to demonstrate differences in salivary metabolic gene expression in oral health and disease based on metatranscriptomic characterization of the salivary microbiota. Future longitudinal studies are warranted to reveal whether salivary screening of metabolic gene expression can identify oral diseases at preclinical stages.

\section{METHODS}

Study population and sample collection

The study population and oral examination has been presented elsewhere in detail. ${ }^{17}$ In brief, 10 patients each, with chronic periodontitis, or manifest dental caries and orally healthy controls were enrolled in April 2015 at Copenhagen University, School of Dentistry. Periodontitis was defined as bleeding on probing $\geq 25 \%$ of total sites + minimum two teeth with clinical attachment level $\geq 4 \mathrm{~mm}+$ minimum two teeth with probing depth $\geq 6 \mathrm{~mm}$ and dental caries was defined as manifest untreated caries $\geq 3$ surfaces. ${ }^{17}$ All 30 participants signed informed consent. The study was completed in accordance with all relevant guidelines and procedures, approved by the regional ethical committee $(\mathrm{H}-15000856)$ and reported to the Danish Dental Authorization (2015-54-0970).

Stimulated saliva samples $(n=30)$ were collected between 8:00 a.m. and 11:00 a.m. according to a standardized protocol. ${ }^{33}$ Immediately after collection each saliva sample was divided in four aliquots of $1 \mathrm{~mL}$ each. Two of these aliquots have previously been used for analysis of bacterial $16 \mathrm{~S}$ metabarcoding targeting rRNA gene ${ }^{17}$ and metaproteomics. ${ }^{21}$ The two remaining aliquots were allocated for this study, one each for metagenomics and metatranscriptomics. RNAlater (Life Technologies, Denmark) was added to the aliquot allocated for metatranscriptomics and all aliquots were immediately stored at $-80^{\circ} \mathrm{C}$ until further processing.

\section{Metagenomic and metatranscriptomic library preparation and sequencing}

Frozen saliva samples were thaw on ice and centrifuged to collect debris. Lysing Matrix B tube (MP Biomedicals) was used to lyse the debris (10 m, $30 \mathrm{~s}$, twice). DNA and RNA (i.e., total RNA) were extracted using MasterPure $^{\text {TM }}$ Complete DNA and RNA Purification Kit (Epientre, Chicago, IL, USA). For DNA samples, RNase was used to remove RNA contamination. For RNA samples, DNase was used to remove DNA contamination.

RNA quality, quantity and residual DNA were determined using a 2100 Bioanalyzer (Agilent Technologies, Santa Clara, CA, USA), as well as Invitrogen RiboGreen and PicoGreen assays (ThermoFisher Scientific, Waltham, MA, USA), respectively. Library preparation was performed according to the TruSeq Stranded mRNA protocol (Illumina, San Diego, CA, USA) with the following modifications: The oligo-dT mRNA purification step was omitted and instead, 200ng of total RNA were directly added to the Elution2-Frag-Prime step. The PCR amplification step, which selectively enriches for library fragments that have adapters ligated on both ends, was performed according to the manufacturer's recommendation but the number of amplification cycles was reduced to 12. Each library was uniquely tagged with one of Illumina's TruSeq LT RNA barcodes to enable library pooling for sequencing. RNA libraries were then sequenced in six lanes of Illumina Hiseq2500 rapid runs at a read-length of $100 \mathrm{bp}$ pairedend, generating an average of $32.71 \mathrm{M}$ reads per sample $(26.40-38.44 \mathrm{M})$.

DNA quality and quantity were determined using a 2100 Bioanalyzer and Invitrogen's PicoGreen assay, respectively. Library preparation was performed according to Illumina's TruSeq Nano DNA Sample Preparation protocol. The samples were sheared on a Covaris S220 (Covaris, Woburn, MA, USA) to $~ 450 \mathrm{bp}$, following the manufacturer's recommendation, and uniquely tagged with one of Illumina's TruSeq LT DNA barcodes. DNA libraries were then sequenced in 2 lanes of an Illumina Hiseq2500 rapid run at a read-length of $250 \mathrm{bp}$ paired-end, generating an average of $25.62 \mathrm{M}$ (16.46-32.64 $\mathrm{M}$ ) reads per sample.

Sequence data was deposited in GenBank (Sequence Read Archive) and is available under the BioProject (PRJNA396840).

\section{Read preprocessing}

Illumina adaptors, $5^{\prime}$ or $3^{\prime}$ bases with quality scores lower than 20 , as well as read pairs having a mate with any ambiguous base (i.e., $\mathrm{N}$ ) or shorter than 150 or $50 \mathrm{bp}$ for DNA and RNA sequences, respectively, were trimmed using cutadapt in paired-end mode (version 1.10, -a -A --max-n 0 -n 1 -q 20,20 --quality-base 33 --minimum-length 150/50 -O 6). Overall, qualitytrimmed reads represented $98.1 \%(97.5-98.6 \%)$ of DNA and $99.3 \%(99.0$ $-99.4 \%)$ of RNA reads.

RNA reads were then subjected to sortmeRNA (version 2.0), ${ }^{34}$ using provided rRNA databases and default parameters for in silico depletion of ribosomal RNA. Ribosomal RNA sequences represented an average of $83.9 \%$ of the quality-trimmed RNA reads (71.1-88.4\%). Human reads were then removed from metagenomic and metatranscriptomic dataset by aligning DNA and mRNA reads to the human genome (h38, ftp://ftp.ncbi. nlm.nih.gov/genomes/all/GCF/000/001/405/GCF_000001405.36_GRCh38. p10/GCF_000001405.36_GRCh38.p10_genomic.fna.gz) using Bowtie2 (version 2.2.9, --un --sensitive-local). Overall, around 9.1 M (2.3-9.2 M) DNA and 4.9 M (2.9-9.1 M) RNA reads were used after quality filtering, rRNA removal and host (i.e., human) contamination for further analysis. Prior to further analysis, $2.26 \mathrm{M}$ reads were randomly selected per sample to obtain equivalent sequencing depth and allow robust comparison.

\section{Taxonomic and functional community profiling}

In order to characterize the taxonomic composition of metagenomic and metatranscriptomic dataset we used a similar approach as llott et al. 2016. ${ }^{35}$ Subsampled sequences were aligned against the NCBI nonredundant (NR) protein database (ftp.ncbi.nlm.nih.gov/blast/db/FASTA/nr. gz, March 2016) using DIAMOND (version 0.7.10.59) ${ }^{36}$ with default parameters. The lowest common ancestor approach implemented in MEGAN6 (L.C.A., version CE_6_5_5), ${ }^{37}$-ms 50 (minimum alignment bitscore $=50)$-supp 0 -sup 25) was used to assign reads at the phylum, genus and species levels for the metagenomic and metatranscriptomic dataset, respectively. In order to characterize the molecular level function of the salivary microbiome, aligned reads were also assigned to KO functional orthologs using GI to KEGG KO functional identifier mapping file as implemented in MEGAN6. ${ }^{37}$

\section{Statistical analysis}

Taxonomic and functional count tables from metagenomic and metatranscriptomic dataset were exported from MEGAN6 and imported as single phyloseq objects (phyloseq, R). Taxonomic and functional tables were filtered to remove taxa accounting less than 100 sequences in total and observed in less than 3 samples (filter_taxa function). Tables were then rarefied to an even sequencing depth using rarefy_even_depth function.

Microbial communities were characterized using alpha-diversity indices (i.e., number of observed taxa and Shannon diversity indices) and betadiversity (i.e., Bray-Curtis dissimilarity) at the Phylum, Genus and Species levels on both metagenomic and metatranscriptomic datasets.

Differences in alpha and beta diversity between healthy control, caries and periodontitis groups dataset were tested using ANOVA and PERMANOVA statistical tests for alpha and beta-diversity, respectively (i.e., Adonis, PERMANOVA functions). Gaussian distribution of ANOVA residuals and multivariate spread was tested to ensure ANOVA and PERMANOVA assumptions (i.e., shapiro.test and betadisper). Statistical significance of taxa-group association was tested using one-sided signassoc function from the indicspecies R-package. Sidak's correction was applied for multiple testing. RNA/DNA log ratios were determined for the relative abundance of taxa identified in metagenomic and metatranscriptomic dataset at phylum and species level. Due to skewed distribution of RNA/DNA log ratios, non-parametric Kruskal-Wallis $H$ test was applied to test differences between groups.

Differential expression analysis of identified KO functional orthologs between healthy controls and patients with periodontitis and caries were conducted on unrarefied functional counts using DESeq2 standard meta-/ transcriptomic pipeline which includes size factor normalization and standardization procedures as well as negative binomial model testing. pvalues were adjusted for multiple testing using FDR correction ${ }^{38}$ - using the following parameters: test = "wald" and fitType = "parametric").

Data availability statement

Sequence data that support the findings of this study have been deposited in GenBank (Sequence Read Archive) and is available under the BioProject (PRJNA396840). 


\section{ACKNOWLEDGEMENTS}

This study was supported by SCELSE core funding.

\section{AUTHOR CONTRIBUTIONS}

Daniel Belstrøm (D.B.), Palle Holmstrup (P.H.), and Michael Givskov (M.G.) designed the study. D.B. and Tim Holm Jakobsen (T.H.J.) collected and stored the samples. Yang Liu (Y.L.), Liang Yang (L.Y.), Daniela I. Drautz-Moses (D.I.D.M.) and Stephan C. Schuster (S.C.S.) performed DNA and RNA sequencing. Florentin Constancias (F.C.), Gurjeet Singh Kohli (G.S.H.) and D.B. performed the bioinformatics analyses. D.B., F.C., and M. G. wrote the manuscript, which P.H., T.H.J., Y.L., L.Y., D.I.D.M., S.C.S., and G.S.H. critically revised. All authors read and approved the final version of the manuscript.

\section{ADDITIONAL INFORMATION}

Supplementary Information accompanies the paper on the npj Biofilms and Microbiomes website (https://doi.org/10.1038/s41522-017-0031-4).

Competing interests: The authors declare that they have no competing financial interests.

Publisher's note: Springer Nature remains neutral with regard to jurisdictional claims in published maps and institutional affiliations.

\section{REFERENCES}

1. Pihlstrom, B. L., Michalowicz, B. S. \& Johnson, N. W. Periodontal diseases. Lancet 366, 1809-1820 (2005).

2. Selwitz, R. H., Ismail, A. I. \& Pitts, N. B. Dental caries. Lancet 369, 51-59 (2007).

3. Paster, B. J. et al. Bacterial diversity in human subgingival plaque. J. Bacteriol. 183, 3770-3783 (2001)

4. Aas, J. A., Paster, B. J., Stokes, L. N., Olsen, I. \& Dewhirst, F. E. Defining the normal bacterial flora of the oral cavity. J. Clin. Microbiol. 43, 5721-5732 (2005).

5. Belda-Ferre, P. et al. The oral metagenome in health and disease. ISME J. 6, 46-56 (2012).

6. Colombo, A. P. et al. Comparisons of subgingival microbial profiles of refractory periodontitis, severe periodontitis, and periodontal health using the human oral microbe identification microarray. J. Periodontol. 80, 1421-1432 (2009).

7. Takahashi, N. \& Nyvad, B. The role of bacteria in the caries process: ecological perspectives. J. Dent. Res. 90, 294-303 (2011).

8. Benitez-Paez, A., Belda-Ferre, P., Simon-Soro, A. \& Mira, A. Microbiota diversity and gene expression dynamics in human oral biofilms. BMC Genomics 15, 311 (2014).

9. Duran-Pinedo, A. E. et al. Community-wide transcriptome of the oral microbiome in subjects with and without periodontitis. ISME J. 8, 1659-1672 (2014).

10. Yost, S., Duran-Pinedo, A. E., Teles, R., Krishnan, K. \& Frias-Lopez, J. Functional signatures of oral dysbiosis during periodontitis progression revealed by microbial metatranscriptome analysis. Genome Med. 7, 27 (2015).

11. Dawes, C. et al. The functions of human saliva: A review sponsored by the World Workshop on Oral Medicine VI. Arch. Oral. Biol. 60, 863-874 (2015).

12. Giannobile, W. V., McDevitt, J. T., Niedbala, R. S. \& Malamud, D. Translational and clinical applications of salivary diagnostics. Adv. Dent. Res. 23, 375-380 (2011).

13. Yoshizawa, J. M. et al. Salivary biomarkers: toward future clinical and diagnostic utilities. Clin. Microbiol. Rev. 26, 781-791 (2013).

14. Schroder, S. A., Bardow, A., Eickhardt-Dalboge, S., Johansen, H. K. \& Homoe, P. Is parotid saliva sterile on entry to the oral cavity? Acta. Otolaryngol. 137, 1-6 (2017).

15. Curtis, M. A., Zenobia, C. \& Darveau, R. P. The relationship of the oral microbiotia to periodontal health and disease. Cell. Host. Microbe 10, 302-306 (2011).

16. Segata, N. et al. Composition of the adult digestive tract bacterial microbiome based on seven mouth surfaces, tonsils, throat and stool samples. Genome Biol. 13, R42 (2012).

17. Belstrom, D., Paster, B. J., Fiehn, N. E., Bardow, A. \& Holmstrup, P. Salivary bacterial fingerprints of established oral disease revealed by the Human Oral Microbe Identification using Next Generation Sequencing (HOMINGS) technique. J. Oral Microbiol. 8, 30170 (2016).
18. Paju, S. et al. Detection of multiple pathogenic species in saliva is associated with periodontal infection in adults. J. Clin. Microbiol. 47, 235-238 (2009).

19. Do, T., Sheehy, E. C., Mulli, T., Hughes, F. \& Beighton, D. Transcriptomic analysis of three Veillonella spp. present in carious dentine and in the saliva of caries-free individuals. Front Cell Infect. Microbiol. 5, 25 (2015).

20. Yang, F. et al. Saliva microbiomes distinguish caries-active from healthy human populations. ISME J. 6, 1-10 (2012).

21. Belstrom, D. et al. Metaproteomics of saliva identifies human protein markers specific for individuals with periodontitis and dental caries compared to orally healthy controls. PeerJ. 4, e2433 (2016).

22. Belstrom, D. et al. Altered bacterial profiles in saliva from adults with caries lesions: A case-cohort study. Caries Res. 48, 368-375 (2014).

23. Belstrom, D. et al. Differences in bacterial saliva profile between periodontitis patients and a control cohort. J. Clin. Periodontol. 41, 104-112 (2014).

24. Luo, A., Yang, D., Xin, B., Paster, B. \& Qin, J. Microbial profiles in saliva from children with and without caries in mixed dentition. Oral. Dis. 18, 595-601 (2012).

25. Heller, D., Silva-Boghossian, C. M., do Souto, R. M. \& Colombo, A. P. Subgingival microbial profiles of generalized aggressive and chronic periodontal diseases. Arch. Oral. Biol. 57, 973-980 (2012).

26. Socransky, S. S., Haffajee, A. D., Cugini, M. A., Smith, C. \& Kent, R. L. Jr. Microbial complexes in subgingival plaque. J. Clin. Periodontol. 25, 134-144 (1998).

27. Torlakovic, L. et al. Microbial community succession on developing lesions on human enamel. J. Oral Microbiol. 4, 1-7 (2012).

28. Kulekci, G., Leblebicioglu, B., Keskin, F., Ciftci, S. \& Badur, S. Salivary detection of periodontopathic bacteria in periodontally healthy children. Anaerobe 14, 49-54 (2008).

29. Relvas, M., Coelho, C., Velazco, H. C. \& Ramos, E. Cariogenic bacteria and dental health status in adolescents: the role of oral health behaviours. Eur. J. Paediatr. Dent. 15, 281-287 (2014).

30. Jenkinson, H. F. \& Lamont, R. J. Oral microbial communities in sickness and in health. Trends Microbiol. 13, 589-595 (2005).

31. Marsh, P. D. Microbiology of dental plaque biofilms and their role in oral health and caries. Dent. Clin. North Am. 54, 441-454 (2010).

32. Nyvad, B., Crielaard, W., Mira, A., Takahashi, N. \& Beighton, D. Dental caries from a molecular microbiological perspective. Caries Res. 47, 89-102 (2013).

33. Kongstad, J. et al. Findings from the oral health study of the Danish health examination survey 2007-2008. Acta. Odontol. Scand. 71, 1560-1569 (2013).

34. Kopylova, E. et al. Deciphering metatranscriptomic data. Methods Mol. Biol. 1269, 279-291 (2015).

35. llott, N. E. et al. Defining the microbial transcriptional response to colitis through integrated host and microbiome profiling. ISME J. 10, 2389-2404 (2016).

36. Buchfink, B., Xie, C. \& Huson, D. H. Fast and sensitive protein alignment using DIAMOND. Nat. Methods 12, 59-60 (2015).

37. Huson, D. H. et al. MEGAN community edition-interactive exploration and analysis of large-scale microbiome sequencing data. PLoS Comput. Biol. 12, e1004957 (2016).

38. Love, M. I., Huber, W. \& Anders, S. Moderated estimation of fold change and dispersion for RNA-seq data with DESeq2. Genome Biol. 15, 550 (2014).

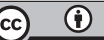

Open Access This article is licensed under a Creative Commons Attribution 4.0 International License, which permits use, sharing, adaptation, distribution and reproduction in any medium or format, as long as you give appropriate credit to the original author(s) and the source, provide a link to the Creative Commons license, and indicate if changes were made. The images or other third party material in this article are included in the article's Creative Commons license, unless indicated otherwise in a credit line to the material. If material is not included in the article's Creative Commons license and your intended use is not permitted by statutory regulation or exceeds the permitted use, you will need to obtain permission directly from the copyright holder. To view a copy of this license, visit http://creativecommons. org/licenses/by/4.0/.

(c) The Author(s) 2017 